\title{
Lower bounds of success probabilities for high-fidelity approach in KLM scheme
}

\author{
Kazuto Oshima* \\ National Institute of Technology, Gunma College, Maebashi 371-8530, Japan
}

\begin{abstract}
In the Knill-Laflamme-Milburn (KLM) scheme, the success probability of quantum teleportation is given by $\frac{n}{n+1}$, wehre $2 n$ is the number of the ancilla qubits. For the high-fidelity approach in the KLM scheme, the success probability is approximately given by $1-\frac{1}{n^{2}}$ for large $n$. We give an explicit prescription to prepare an optimal ancilla state and give an exact lower bound of the success probability for the high-fidelity approach for arbitrary $n$.
\end{abstract}

PACS number(s): 03.67.Lx

*E-mail: oshima@nat.gunma-ct.ac.jp 


\section{Introduction}

Quantum teleportation[1] is the most important technique in quantum computation and quantum communication. Cluster state quantum computation [2] is a useful application of quantum teleportation. Photon is expected be one of the most promising media for quantum information. Using photons as qubits is advantageous in the points that it is robust against noise and it is easily transmitted. It is, however, difficult to perform two-qubits gates between two photonic qubits. Knill, Laflamme and Milburn(KLM) 3] have invented a scheme to carry out quantum teleportation of a photonic qubit with the probability near to 1 . Combining the KLM scheme and the strategy of Gottesman and Chuang [4], we can perform two-qubits gates between two photonic qubits with the probability near to 1 . Nearly deterministic two-qubits gates are useful in connecting cluster states into arbitrary shapes as well as in universal quantum computation. In the KLM scheme, to boost the success probability close to 1 , we should prepare a large number of ancilla qubits. This causes the difficulty that that the number optical resources such as beam splitters and phase shifters increases. Optimality about ancilla states have been studied by Grudka and Modlawska [5] for the purpose of perfect quantum teleportation.

Franson et. al. [6] have proposed a new line of approach (high-fidelity approach) in the KLM scheme. Preparing ancilla states properly, they have shown that the success probability, in the sense of fidelity, approaches to 1 faster than the original setting as the number of ancilla qubits increases. Their approach is useful in saving the resources. Their analysis, however, uses approximation valid when the number of ancilla qubits is sufficiently large. The purpose of this paper is to give an explicit prescription for preparing an optimal ancilla state for any number of ancilla qubits. This ancilla state gives a lower bound of the success probability of quantum teleportation without approximation in the sense of fidelity.

\section{Ancilla states and success probabilities}

We prepare a $2 n$-qubits ancilla state as $\left|u_{n}\right\rangle=\sum_{i=0}^{n} f(i)|0\rangle^{n-i}|1\rangle^{i}|0\rangle^{i}|1\rangle^{n-i}$, where $|0\rangle^{i}$ means $i$ photons in the state $|0\rangle$ etc. and $f(i)^{\prime}$ 's are real coefficients normalized as $\sum_{i=0}^{n} f(i)^{2}=1$. In the original KLM scheme they are settled as $f(i)=\frac{1}{\sqrt{n+1}}, i=0,1, \cdots n$. We consider to teleport a quantum state $|\psi\rangle=\alpha|0\rangle+\beta|1\rangle,|\alpha|^{2}+|\beta|^{2}=1$. We perform $n+1$-poin quantum Fourier transformation $\hat{F}_{n+1}$ on the state $|\psi\rangle$ and the first $n$ qubits in the ancilla state. Suppose we observe $k(0 \leq k \leq n+1)$ photons after the transformation. When $k=0$ and $k=n+1$ we fail in the procedure. In another case, we obtain the teleported state

$$
\left|q_{k}\right\rangle=\frac{\alpha f(k)|0\rangle+\beta f(k-1)|1\rangle}{\sqrt{|\alpha|^{2} f(k)^{2}+|\beta|^{2} f(k-1)^{2}}}
$$

at the $k$-th qubit in the latter half of the ancilla qubits. To obtain the state $\left|q_{k}\right\rangle$ in the form of Eq.(1), we should perform certain relative phase shift between the states $|0\rangle$ and $|1\rangle$ depending on the observed $k$-photon state. The probability $p_{k}$ to obtain the state $\left|q_{k}\right\rangle$ is given by

$$
\left.p_{k}=\Sigma_{k}\left|\left\langle k\left|\hat{F}_{n+1}\right| \psi\right\rangle\right| u_{n}\right\rangle\left.\right|^{2}=|\alpha|^{2} f(k)^{2}+|\beta|^{2} f(k-1)^{2},
$$


where the summation about $k$ runs over all possible $k$-photon states and we have used $\Sigma_{k}|k\rangle\langle k|=\hat{I}_{k}$ with $\hat{I}_{k}$ the identity operator on any $k$-photon state. In the high-fidelity approach the success probability $p$ is defined by the expectation value of the square of the fidelity $\left|\left\langle\psi \mid q_{k}\right\rangle\right|^{2}, k=1,2, \cdots, n$; we define $p$ as $p=\sum_{k=1}^{n} p_{k}\left|\left\langle\psi \mid q_{k}\right\rangle\right|^{2}$.

First, let us choose the original state $|\psi\rangle$ as $|\psi\rangle=|+\rangle=\frac{1}{\sqrt{2}}(|0\rangle+|1\rangle)$. In this case the fidelity $F_{k}$ is given by

$$
F_{k}=\left|\left\langle+\mid q_{k}\right\rangle\right|=\frac{1}{\sqrt{2}} \frac{f(k)+f(k-1)}{\sqrt{f(k)^{2}+f(k-1)^{2}}}
$$

and the success probability $p$ is given by

$$
p=\frac{1}{4} \sum_{k=1}^{n}(f(k)+f(k-1))^{2} .
$$

The maximum value of $p$ is given by the largest eigenvalue $\lambda_{n}$ of the following $(n+1) \times$ $(n+1)$ matrix

$$
A=\frac{1}{4}\left(\begin{array}{cccccc}
1 & 1 & 0 & \ldots & 0 & 0 \\
1 & 2 & 1 & \ddots & 0 & 0 \\
0 & 1 & 2 & \ddots & 0 & \vdots \\
\vdots & \ldots & \ddots & \ddots & 1 & 0 \\
0 & \ldots & \ldots & 1 & 2 & 1 \\
0 & \ldots & \ldots & 0 & 1 & 1
\end{array}\right)
$$

This matrix has recently appeared in the literature[7] in the context of port-based teleportation scheme [8]. The corresponding normalized eigenvector of $A$ gives the optimal coefficients

$$
(f(0), f(1), \cdots, f(n)) \text {. }
$$

Note that the condition $f(0)=f(n)$ holds, on account of the symmetric properties of the matrix $A$.

Second, we consider an arbitrary quantum state $|\psi\rangle=\alpha|0\rangle+\beta|1\rangle$. Having settled the coefficients $f(k)$ 's as above, the success probability for the state $|\psi\rangle=\alpha|0\rangle+\beta|1\rangle$ is giben by

$$
p=\sum_{k=1}^{n}\left(|\alpha|^{2} f(k)+|\beta|^{2} f(k-1)\right)^{2} .
$$

It is easy to see that the success probability $p$ takes its minimum value $\lambda_{n}$ at $|\alpha|^{2}=|\beta|^{2}=\frac{1}{2}$ as far as the condition $f(0)=f(n)$ is satisfied. This means that the success probability $p$ is bounded from below by $\lambda_{n}$ for arbitrary quantum state $|\psi\rangle=\alpha|0\rangle+\beta|1\rangle$. For references, we exhibit the value $\lambda_{n}$ and the corresponding coefficients $f(k)$ 's for some small $n$ 's; $\lambda_{2}=$ $\frac{3}{4},(f(0), f(1), f(2))=\frac{1}{\sqrt{6}}(1,2,1) ; \lambda_{3}=\frac{2+\sqrt{2}}{4},(f(0), f(1), f(2), f(3))=\frac{1}{\sqrt{8+4 \sqrt{2}}}(1,1+$ $\sqrt{2}, 1+\sqrt{2}, 1) ; \lambda_{4}=\frac{5+\sqrt{5}}{8},(f(0), f(1), f(2), f(3), f(4))=\frac{1}{\sqrt{16+5 \sqrt{5}}}\left(1, \frac{3+\sqrt{5}}{2}, 1+\sqrt{5}, \frac{3+\sqrt{5}}{2}, 1\right)$. It will be not so hard to see that $\lambda_{n}$ approaches to 1 as $\lambda_{n}=\frac{1}{2}+\frac{1}{2} \cos \frac{\pi}{n+1}$ as $n$ increases. 
The largest eigenvalue $\lambda_{n}$ can also be written as $\lambda_{n}=\frac{1}{2}+\frac{1}{4} \mu_{n}$, where $\mu_{n}=2 \cos \frac{\pi}{n+1}$ is the largest eigenvalue of the following simple $n \times n$ matrix $B$ :

$$
B=\left(\begin{array}{cccccc}
0 & 1 & 0 & \ldots & 0 & 0 \\
1 & 0 & 1 & \ddots & \vdots & 0 \\
0 & 1 & 0 & \ddots & 0 & \vdots \\
\vdots & \ddots & \ddots & \ddots & 1 & 0 \\
0 & \ldots & \ddots & 1 & 0 & 1 \\
0 & \ldots & \ldots & 0 & 1 & 0
\end{array}\right)
$$

An expectation value of fidelity, not the square of fidelity, has already been computed in closed form in the port-based teleportation scheme, which is somewhat different from the present one, with an elaborate method[9].

\section{Conclusions}

We have given the exact lower bound of the success probability of quantum teleportation for high-fidelity approach in the KLM scheme. Our result is valid for any number of ancilla qubits and is of practical use. We have given the prescription to prepare the most preferable anncilla state for any number of ancilla qubits.

\section{Acknowledgment}

The author thanks Kenta Takahashi and Yuya Tamura for useful discussion. The author thanks Sergii Strelchuk for helpful advice. 


\section{References}

[1] C.H.Bennett, G.Brassard, C.Crépeau, R.Jozsa, A.Peres and W.K.Wootters, Phys.Rev.Lett.70, 1895(1993).

[2] R.Raussendorf and H.J.Briegel, Phys.Rev.Lett., 86, 5188(2001); H.J.Briegel and R.Raussendorf, Phys.Rev.Lett., 86, 910(2001).

[3] E.Knill, R.Laflamme and G.J.Milburn, Nature, 409, 46(2001).

[4] D.Gottesman and I.L.Chuang, Nature, 402, 390(1999).

[5] A.Grudka and J.Modlawska, Phys.Rev.A77, 01431(2008).

[6] J.D.Franson, M.M.Donegan, M.J.Fitch, B.C.Jacobs and T.B.Pittman, Phys.Rev.Lett., 89, 137901(2002).

[7] M. Mozrzymas, M. Studziński, S. Strelchuk and M. Horodecki, arXive: quant-ph, 1707.08456, (2017).

[8] S. Ishizaka and T. Hiroshima, Phys.Rev.Lett. 101, 240501(2008).

[9] S. Ishizaka and T. Hiroshima, Phys.Rev.A.79, 042306(2009). 